\title{
Age-related structural-mechanical property changes in human peroneus longus muscle
}

\author{
A. Niyomchan ${ }^{1}$, B. Panichareon ${ }^{1}$, A. Siriphorn ${ }^{2}$, T. Wongtawatchai ${ }^{3}$ \\ ${ }^{1}$ Department of Anatomy, Faculty of Medicine Siriraj Hospital, Mahidol University, Bangkok, Thailand \\ ${ }^{2}$ Department of Physical Therapy, Faculty of Allied Health Sciences, Chulalongkorn University, Bangkok, Thailand \\ ${ }^{3}$ Department of Anatomy, Faculty of Science, Prince of Songkla University, Hat Yai, Songkhla, Thailand
}

[Received: 31 August 2018; Accepted: 8 October 2018]

\begin{abstract}
Background: Functional impairment of the muscle-tendon unit is one of the most remarkable effects of aging. The function of the peroneus longus muscle is to stabilise the foot and ankle joint. A deterioration of the structural and mechanical properties of this muscle can potentially lead to foot problems in older adults. This study aimed to investigate the effects of age on structural, histological, and mechanical features in peroneus longus muscle samples taken from embalmed cadavers of two different age groups; young adult (30-60 years) and old adult (over 60 years). Materials and methods: The mechanical property was analysed through the results of cross-sectional area, tensile, tensile stress, and modulus of elasticity. The arrangement of the collagen in the perimysium and tendon was examined by scanning electron microscopy. Fatty infiltration within the musculotendinous junction was evaluated by Masson' trichrome stained muscle sections.

Results: This study thus provides evidence that there are indeed age-induced mechanical property changes in the peroneus longus muscle, which include reductions in the tensile force, tensile stress, and modulus of elasticity, and is related to the malformation of collagen fibrils and the massive fat accumulation in the musculotendinous junction.

Conclusions: These alterations may further result in a reduction of muscle strength and quality in an older person. (Folia Morphol 2019; 78, 2: 401-407)
\end{abstract}

Key words: aging, collagen, musculotendinous junction, tendon

\section{INTRODUCTION}

Aging, a gradual deterioration of the physiological processes associated with a dramatic decrease in muscle-tendon performance $[27,28]$. A reduction in muscle strength and mechanical properties of tendons often leads to disability and loss of mobility in older adults [10]. One of the most extensively utilised parts of the body is the foot, which is primarily responsible for weight-bearing activities. Muscles and tendons, aside from bones, are responsible for controlling the normal position and function of the foot. Changes in structural and mechanical properties of the musculotendinous unit can cause foot problems, a common concern for older adults [24]. Indeed, among the many muscles and tendons in the foot, the tibialis posterior and peroneus longus have essential roles. The tibialis posterior is the strong supporter of the medial longitudinal arch and assists in plantar flexion and inversion [11, 13], while the peroneus longus produces eversion and plantar flexion. Furthermore, it is also responsible for stabilising two longitudinal arches (medial and lateral arches) [14, 25]. As such, both of these muscles affect any dynamic changes involved in foot alignment [14]. Tibialis posterior dysfunction is well recognised as a cause of flatfeet 


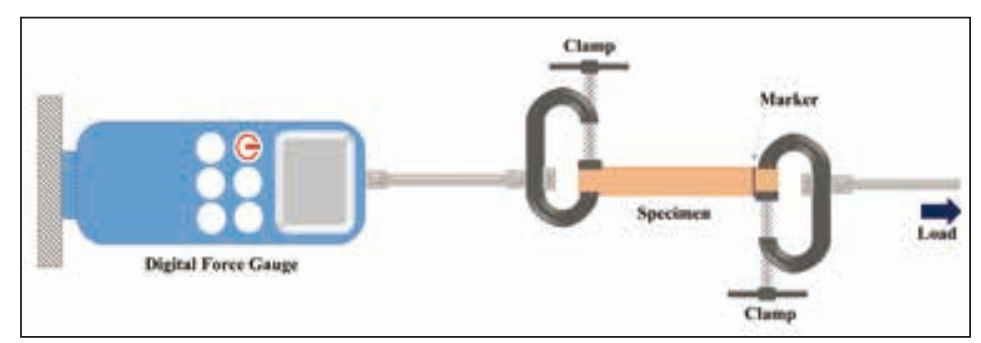

Figure 1. The schematic drawing representing the set up used for testing mechanical properties of peroneus longus in adult and older adult cadavers through the use of a multi-functional digital force gauge.

in both adults and the elderly [13]. Thus, many studies often investigate the alterations of this muscle during the development of aging [3,34]. However, there has been no comprehensive evaluation of degenerative changes in structural and mechanical properties of the peroneus longus muscle to date. Accordingly, the purpose of this study was thus to examine aging-induced alterations involved in the structural and mechanical properties of the peroneus longus which can control foot posture along with the other foot muscles. While the morphological alterations observed in the present study may be one mechanism in the age-related structural, functional changes of muscle-tendon complex and new information will grant a clear awareness of the effects of peroneus longus degradation in an older person.

\section{MATERIALS AND METHODS}

\section{Specimen collection}

The lower extremities of 10 adult (30-60 years) and 10 older adult (over 60 years) embalmed cadavers consisting of both genders were obtained for the dissection of the peroneus longus, including muscular part, musculotendinous junction and tendon portion from both sides of legs. Peroneus longus muscles from the right legs were used for the mechanical testing, while the muscle from the left side were collected for histological and structural evaluations through light and scanning electron microscopic (SEM) studies. The cadavers had been donated to the Department of Anatomy, Faculty of Medicine Siriraj Hospital, Mahidol University for use in anatomical studies. The embalming was done within $24 \mathrm{~h}$ of death using a formalin that was mixed with adjuvants. The embalmed cadavers were kept and preserved for approximately 1 year prior used for analysis. Ethical approval for this study (COA no. Si074/2561) was granted by Institutional Review Board, Faculty of Medicine Siriraj Hospital, Mahidol University.

\section{Mechanical property testing}

After the peroneus longus muscle was dissected out from the cadaver, the highest point of musculotendinous junction was identified and marked with a permanent marker. The specimen was then cut into a 90-mm long section with a 70-mm long tendon side and 20-mm long muscle fibre side. Both free ends of the muscles were then clamped with adjustable C-clamps. The specimen was then placed in a multi-functional digital force gauge IPX-800 (INSPEX ${ }^{\circledR}$, Bowers Group, United Kingdom) with the side of the tendon facing the force gauge (Fig. 1). The validity and reliability were analysed by four known weights and test-retest values of four specimens, respectively. This system was found to be valid (per cent accuracy: $\pm 1.9 \%)$ and reliable $\left(\mathrm{ICC}_{3,1}=0.93\right)$. Prior to mechanical testing, the specimen was tightened to allow for viscoelastic stress relaxation. The force gauge was then calibrated to zero. Subsequently, an axial tensile force was applied to the muscle fibre side until it reached a 1-mm displacement of the musculotendinous junction. A maximum force in a unit of Newtons $(\mathrm{N}$ ) was recorded for each specimen.

After that, the specimen was removed from the clamps and then cross-sectionally dissected at the musculotendinous junction. Photographs of the cross-sectional area (CSA) of each specimen were obtained using a digital camera. The UTHSCSA ImageTool for Windows version 3.0 (University of Texas Health Science Centre in San Antonio, USA) was used for measuring the CSA in a unit of square millimeter $\left(\mathrm{mm}^{2}\right)$. The following formula was then used to calculate the tensile stress in a unit of Megapascals (MPa): Tensile stress $(\mathrm{MPa})=$ Force $(\mathrm{N}) / \mathrm{CSA}$ $\left(\mathrm{mm}^{2}\right)$. The modulus of elasticity (Young's modulus) was also calculated in a unit of MPa through the following formula: modulus of elasticity $(\mathrm{MPa})=$ Tensile stress $(\mathrm{MPa}) /$ / [amount of length change $(\Delta \mathrm{L})$ / original length $\left(\mathrm{L}_{0}\right)$ ].

\section{Histological study}

The musculotendinous parts of the peroneus longus muscles were fixed in an immersion of 10\% formalin, dehydrated in ascending grades of alcohol, cleared in xylene, infiltrated and embedded in paraffin. The paraffin block was cut by a rotatory microtome (Leica RM2035, Nussloch, Germany) with $6 \mu \mathrm{m}$ thickness. Sections were 
Table 1. Mean \pm standard deviation of the mechanical properties of peroneus longus under loading condition for the displacement of the musculotendinous junction for 1-mm length in adult and older adult

\begin{tabular}{lccc}
\hline Properties & Adult $(\mathbf{n}=\mathbf{1 0})$ & Older adult $(\mathbf{n}=\mathbf{1 0})$ & $\mathbf{P}$ \\
\hline Tensile (N) & $45.60 \pm 17.69$ & $28.13 \pm 7.62^{*}$ & $0.010^{\mathrm{a}}$ \\
Cross-sectional area $\left[\mathrm{mm}^{2}\right]$ & $106.80 \pm 47.34$ & $94.71 \pm 14.35$ & $0.450^{\mathrm{b}}$ \\
Tensile stress (MPa) & $0.47 \pm 0.19$ & $0.30 \pm 0.10^{*}$ & $0.025^{\mathrm{b}}$ \\
Modulus of elasticity (MPa) & $32.64 \pm 12.97$ & $21.35 \pm 6.89^{*}$ & $0.026^{\mathrm{b}}$ \\
\hline
\end{tabular}

${ }^{*}$ Significant difference $(p<0.05)$; ${ }^{a}$-value was calculated by the Mann-Whitney test; ${ }^{b} p$-value was calculated by unpaired $t$-test

stained with Masson's trichrome and analysed under an Olympus Bx43 microscope. The pictures were taken by a DP73 digital camera attached to the CellSens Standard software (Olympus Optical, Co. Ltd., Tokyo, Japan).

\section{Scanning electron microscopy sample preparation}

The muscular and tendon portion of the peroneus longus muscles were cut into small cubes with a size of $0.5 \times 0.5 \times 0.2 \mathrm{~cm}$. The specimens were washed in a $0.1 \mathrm{M}$ phosphate buffer solution at $\mathrm{pH} 7.4$ and postfixed with $1 \%$ osmium tetroxide for $1 \mathrm{~h}$. After washing it with distilled water, the specimens were dehydrated in a graded series of alcohol. The samples were then dried in the critical point dryer using liquid $\mathrm{CO}_{2}$ and mounted on metal stubs. Finally, they were coated with gold-palladium in the sputter-coater and analysed in the JSM-6510LV (Jeol Ltd, Tokyo, Japan) SEM at 15 kV.

\section{Statistical analysis}

Data analysis was performed using the GraphPad Prism programme for Windows version 6.05 (GraphPad software Inc. CA, USA). All the data were presented as mean \pm standard deviation. Prior to performing any between-group comparisons, all properties of the peroneus longus under loading condition were tested for normality using the Shapiro-Wilk test. Three parameters, i.e., CSA, tensile stress, and modulus of elasticity, passed the normality test. Thus, an unpaired t-test was used to compare between the groups for these parameters. In contrast, the Mann-Whitney test was used for the tensile parameter, which did not distribute normally. A p-value level of less than 0.05 was considered to be significant.

\section{RESULTS}

Effect of aging on muscle-tendon mechanical properties

Table 1 shows the results of the between-group comparisons of all parameters. No significant difference in the CSA of peroneus longus musculotendinous junction was found between the adult and older adult. Interestingly, it was apparent from Table 1 that the tensile, tensile stress and elastic modulus of the peroneus longus in older adult were significantly lower than the younger ones.

\section{Effect of aging on muscle-tendon histostructural changes}

The SEM analysis of the peroneus longus muscle fibres revealed that there were long cylindrical forms with cross-striations of myofibrils on the muscle surface. The fibres ran parallel to each other, and they were not branched. However, there were no differences in the muscular morphologic features such as diameter and shape between both groups of cadavers (Fig. 2).

Considerable alterations in the collagen bundles of the perimysium that were overlying muscle fibres were noted in the older adult cadaver. Usually, perimysium comprised of the dense weave collagen bundles. However, the peroneus longus muscle of the older adult cadaver contained thin and loosely packed perimysial collagen bundles, which was comparatively different to the thicker and more compact surface seen in the adult cadaver's perimysial collagen (Fig. 3).

Within the tendon, the normal collagen fibres in the peroneus longus tendon of the young cadavers showed long, straight and tightly packed collagen fibres in a parallel arrangement along the long axis of the tendon (Fig. 4A, C). At higher magnification, most of the collagen fibres appeared to be more crimp and of a helical pattern (Fig. 4E). In an older adult cadaver, the tendon consisted of disorientated and frayed collagen fibres (Fig. 4B). In some tendons, disorganisation and fragmentation of collagen fibrils were observed (Fig. 4D). They were flat, and the fracture surfaces appeared to look like tattered rope. Furthermore, the collagen fibrils appeared straight without helical coils (Fig. 4F).

Light microscopy of the muscle-tendon junction of the peroneus longus muscle showed remarkable changes in the aging process, particularly at the end of muscle inserted into the tendon via the myotendinous junction. In this region, the abundant tendon fibroblasts were embedded in the collagen fibres, and the fibres of collagen were continuously anchored to the terminal extensions of muscle fibres, as shown in the adult cadaver 

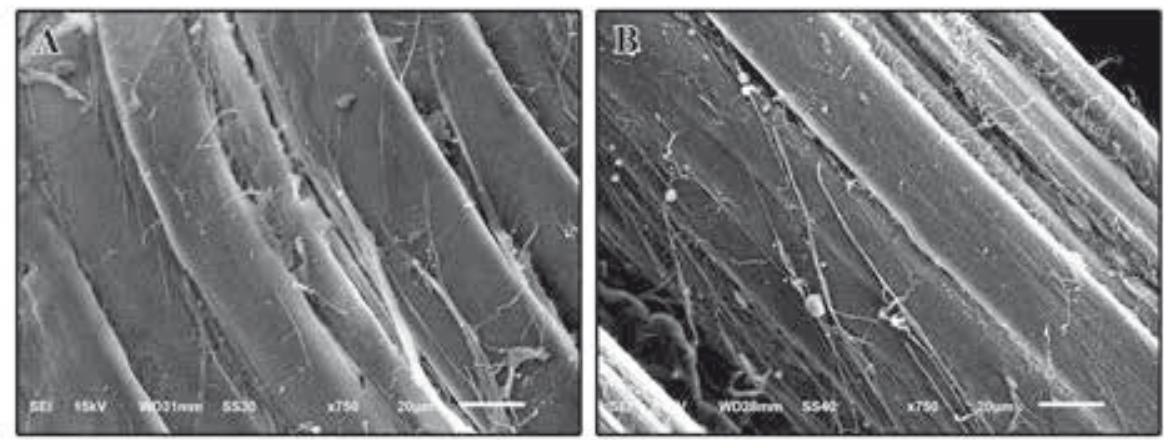

Figure 2. Scanning electron micrographs of the peroneus longus muscle fibres in an adult cadaver (A) and older adult cadaver (B). No differences were observed between younger and older cadavers in their muscle fibre morphology. The muscle fibres were arranged in a simple parallel fashion, with striations running perpendicular to the long axis of the muscle fibre.
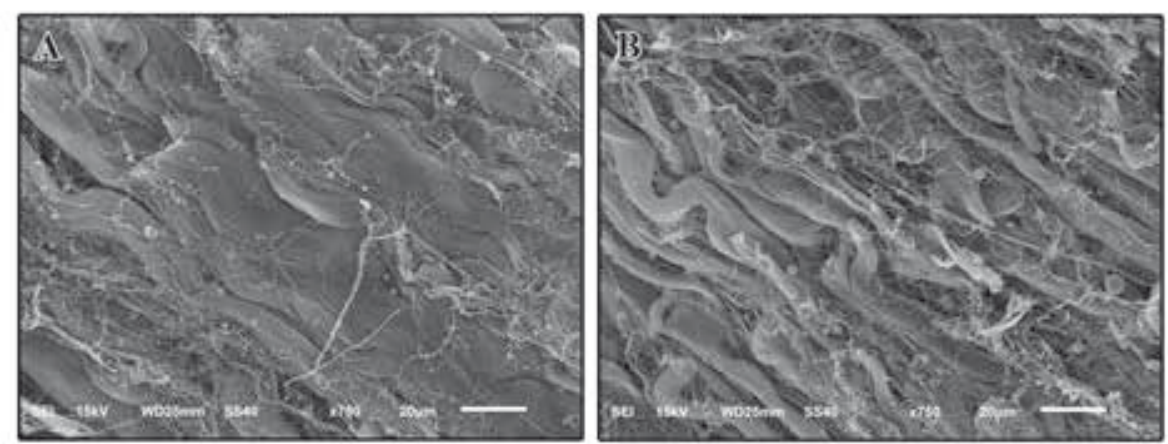

Figure 3. Scanning electron micrographs of the perimysial collagen bundles of peroneus longus muscle in adult cadaver (A) and older adult cadaver (B). A looser arrangement of small perimysial collagen bands was present in the older adult cadaver when compared to the large and tight collagen bundles of perimysium found in the adult cadaver.

(Fig. 5A). With age, it was seen that fat accumulation occurred in the myotendinous junction, and more fats were deposited closer to this region (Fig. 5B).

\section{DISCUSSION}

The peroneus longus, which is comprised of muscular and tendinous tissues, helps to stabilise the arch and assists in stabilising the ankle and foot [23]. It is predominantly active during the stance phase of the gait $[19,21]$, which produces plantarflexed first ray of the foot and also acts as a secondary plantarflexor of the ankle [20]. Thus, deteriorated physical ability of the peroneus longus can contribute to the development of foot deformity $[2,29]$. Age-related changes in the structural and mechanical properties of the muscle-tendon unit further reflect the alterations in functional competencies. Consequently this study investigated the potential architectural and mechanical changes that occur with age in the peroneus longus musculotendinous unit, which could be associated with the increased rate of foot problem cases found clinically in older adults. We examined three different parts of the peroneus longus, including the muscular part, the musculotendinous junction, and tendon portion of young and old adult cadavers. The three main findings of this study were as follows: 1) mechanical properties including tensile, tensile stress and modulus of elasticity were lower, without a change in CSA in old adult cadavers when compared with the younger subjects, 2) collagen fibre disorganisation appeared in the aged tendon and perimysium, and 3) accumulation of fat was noted within the musculotendinous junction.

Mechanical property testing in the present study demonstrated a decline in tensile, tensile stress, and modulus of elasticity of the peroneus longus in old adult cadavers. These changes were linked to alterations that were sustained by the structure of the tendon portion of this muscle. An abnormal architectures of collagen, the chief component in the tendon, were found with aging. The tendon section of the peroneus longus in the aged specimens appeared to be disorganised and fragmented, along with having elongation without crimp. The reasons for these results were age-related alterations in the matrix contents, including an increase in the degradation of collagens and proteoglycans via an activation of matrix metalloproteinases $[5,36]$ and a reduction in intra-molecular cross-links involving in crimp-like structure, a result of advanced glycation endproduct accumulation in advancing age $[1,4]$. 

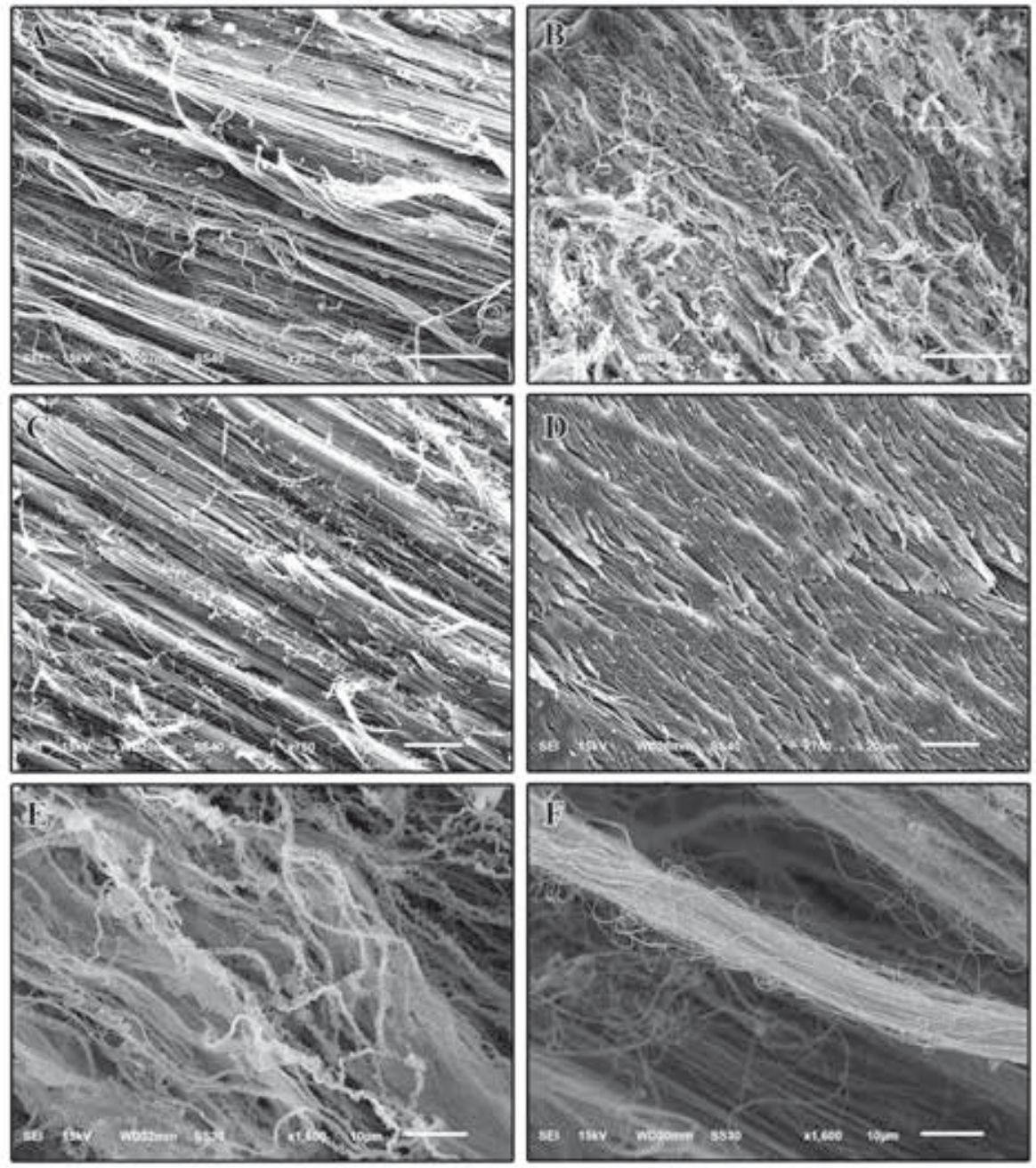

Figure 4. Scanning electron micrographs of the peroneus longus tendon of an adult cadaver (A, C, E) and elderly cadaver (B, D, F). High-density collagen fibres in a parallel alignment $(\mathbf{A})$, thick linear collagen bundles $\mathbf{( C )}$ and coiled collagen fibrils in the tendon $(\mathbf{E})$ were shown in the adult cadaver, whereas the collagen found in the tendon of the elderly appeared disorganised (B), fragmented (D) and had straight collagen fibrils (F).
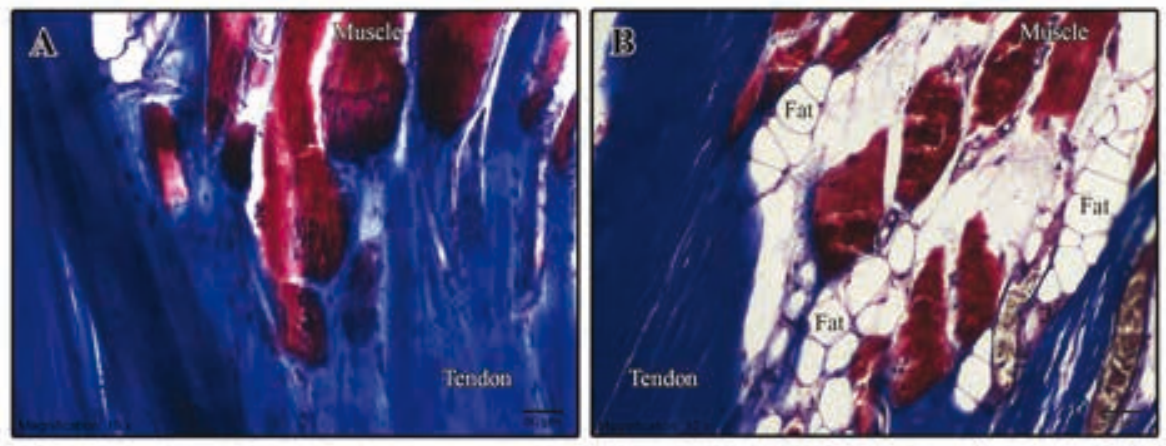

Figure 5. Light micrographs of the peroneus longus muscle at the myotendinous junction in the adult cadaver (A) and elderly cadaver (B). Fats intervened between the terminal end of each muscle fibre and the collagen of the tendon at the myotendinous junction, and a large amount of fat was found in this area in the older adult cadaver, a stark contrast when compared to the closely packed muscle-tendon interdigitation in the adult cadaver. Masson's trichrome staining: blue collagen, red muscle fibre, and black cell nuclei. 
Since each extracellular matrix layer (epimysium, perimysium, endomysium) was primarily dominated by collagen type I and III, which was similar to that in the tendon [12]. The alterations of perimysial collagen arrangement reported in this study were thought to be due to the same mechanism of having increased metalloproteinases regulatory pathway that were used to break down the collagen fibrils, leading to small and loose arranged perimysial collagen bundles in elderly cadavers.

Although the morphology of the muscular part of the peroneus longus remained stable during aging with unchanged shape and size, fat infiltration in the musculotendinous junction was found in the present study. Many previous studies have been reported to be agerelated, but do not appear to factor in sex-related loss of muscle mass, as evidenced by a decrease in muscle fibre CSA and fibre number determining in the various muscles from humans and cadaveric specimens [26, 30]. However, our findings contradict these previous reports. We found that there was no statistical difference in CSA (measure of muscle fibre size) of the peroneus longus between young and old adult cadavers and the muscular architecture did not change substantially with age. The differences between these findings may be due to diversity in the range and distribution of muscle fibre size in the aging, based on types of muscle fibres. Atrophy of type II, fast-twitch or white muscle fibres and hypertrophy in type I, slow-contracting or red fibres can occur in the elderly [7]. Furthermore, a decline in protein synthesis and an enhancement of overall protein degradation rates could also cause the atrophy of muscles [17], while muscle hypertrophy takes place as a compensatory response to the reduction in muscle mass that occurs with aging $[7,18]$. Hepple et al. [7] indicated a variable alteration in the mean muscle fibre CSA of the gastrocnemius muscle. They exhibited a decrease in fibre size in the white region of the gastrocnemius, but increased fibre size in the red region of aged animals. Similar to the animal study, muscle biopsies of the vastus lateralis from humans revealed a greater reduction in muscle fibre size of type II fibres than type I fibres [6]. However, the current study did not find a difference in the mean CSA of the peroneus longus, which is a hybrid muscle fibres containing $62.5 \%$ slow-twitch type I fibres and $37.5 \%$ type II fast-twitch fibres, between young and old cadaveric specimens. Kano et al. [9] similarly reported no statistical difference in the mean CSA of soleus that consisted mainly of type I fibres between young and old rats.

The current study was the first finding to demonstrate fat infiltration within the musculotendinous junction of the old age muscle tissues, as of the previ- ous studies have only reported an accumulation of fat within the muscle and near the myotendinous junction in an advanced age $[22,35]$. An altered lipid metabolism with increasing age could be used to explain the fatty changes in aging muscles through an elevation of cholesterol and triglyceride levels [15], an increase in lipogenesis and an impairment of lipolysis [31] as well as changes in fat distribution from subcutaneous locations to intra-abdominal visceral depots and to harmful ectopic sites [8]. Thus, an ectopic fat deposition within the musculotendinous junction, which was found in this study, might impair muscle-tendon junction, especially the force transmission from muscle fibres to bone via the myotendinous junction and tendon. Moreover, both the perimysium and endomysium play a crucial role in the transmission of force via the action of collagen [16]. Therefore, an accumulation of fat in the musculotendinous junction and structural changes of collagen in the tendon and perimysium during aging caused the peroneus longus muscle to alter its mechanical function. Changes in structural-mechanical properties of the peroneus longus muscle might increase the risk for an abnormal foot shape and foot discomfort in elderly people.

Although the embalmed cadavers can be used for mechanical testing in both soft and hard tissues [32, 33]. A limitation of our study was the use of embalmed cadavers that would have the effect of sample preservation. In future, it would be interesting to confirm these findings using soft and fresh frozen cadavers.

\section{CONCLUSIONS}

In summary, this study suggested age-related changes in the peroneus longus muscle, particularly structural abnormalities in the collagen fibres and fatty infiltration in the musculotendinous junction. Consequently, the tensile, tensile stress, and modulus of elasticity were decreased. These changes might also predict a decrease in the strength and poorer functional outcomes of this muscle.

\section{Acknowledgements}

This work was partially supported by Chalermprakiat Grant, Faculty of Medicine Siriraj Hospital, Mahidol University to A. Niyomchan and B. Panichareon.

The authors gratefully acknowledge Mr. Kasem Koedpuech, Mrs. Nusara Chomanee and Miss Hataichanok Pimpui for the skilful technical help of light and scanning electron microscopy.

\section{REFERENCES}

1. Avery NC, Bailey AJ. Enzymic and non-enzymic cross-linking mechanisms in relation to turnover of collagen: relevance 
to aging and exercise. Scand J Med Sci Sports. 2005; 15(4): 231-240, doi: 10.1111/j.1600-0838.2005.00464.x, indexed in Pubmed: 15998340.

2. Brandes $C B$, Smith RW. Characterization of patients with primary peroneus longus tendinopathy: a review of twenty-two cases. Foot Ankle Int. 2000; 21(6): 462-468, doi: 10.1177/107110070 002100602, indexed in Pubmed: 10884103.

3. Bubra PS, Keighley G, Rateesh S, et al. Posterior tibial tendon dysfunction: an overlooked cause of foot deformity. J Family Med Prim Care. 2015; 4(1): 26-29, doi: 10.4103/2249-4863.152245, indexed in Pubmed: 25810985.

4. Couppé $C$, Hansen P, Kongsgaard M, et al. Mechanical properties and collagen cross-linking of the patellar tendon in old and young men. J Appl Physiol (1985). 2009; 107(3): 880-886, doi: 10.1152/ japplphysiol.00291.2009, indexed in Pubmed: 19556458.

5. Dudhia J, Scott CM, Draper ERC, et al. Aging enhances a mechanically-induced reduction in tendon strength by an active process involving matrix metalloproteinase activity. Aging Cell. 2007; 6(4): 547-556, doi: 10.1111/j.1474-9726.2007.00307.x, indexed in Pubmed: 17578513.

6. Grimby G, Danneskiold-Samsøe B, Hvid K, et al. Morphology and enzymatic capacity in arm and leg muscles in 78-81 year old men and women. Acta Physiol Scand. 1982; 115(1): 125-134, doi: 10.1111/ j.1748-1716.1982.tb07054.x, indexed in Pubmed: 7136797.

7. Hepple RT, Ross KD, Rempfer AB. Fiber atrophy and hypertrophy in skeletal muscles of late middle-aged Fischer $344 \times$ Brown Norway F1-hybrid rats. J Gerontol A Biol Sci Med Sci. 2004; 59(2): 108-117, indexed in Pubmed: 14999023.

8. Hughes VA, Roubenoff R, Wood M, et al. Anthropometric assessment of 10-y changes in body composition in the elderly. Am J Clin Nutr. 2004; 80(2): 475-482, doi: 10.1093/ajcn/80.2.475, indexed in Pubmed: 15277173.

9. Kano $\mathrm{Y}$, Shimegi S, Furukawa $\mathrm{H}$, et al. Effects of aging on capillary number and luminal size in rat soleus and plantaris muscles. J Gerontol A Biol Sci Med Sci. 2002; 57(12): B422-B427, indexed in Pubmed: 12456732.

10. Keller K, Engelhardt M. Strength and muscle mass loss with aging process. Age and strength loss. Muscles Ligaments Tendons J. 2013; 3(4): 346-350, indexed in Pubmed: 24596700.

11. Kitaoka HB, Luo ZP, An KN. Effect of the posterior tibial tendon on the arch of the foot during simulated weightbearing: biomechanical analysis. Foot Ankle Int. 1997; 18(1): 43-46, doi: 10.1177/107 110079701800109, indexed in Pubmed: 9013114

12. Kjaer M. Role of extracellular matrix in adaptation of tendon and skeletal muscle to mechanical loading. Physiol Rev. 2004; 84(2): 649-698, doi: 10.1152/physrev.00031.2003, indexed in Pubmed: 15044685.

13. Kohls-Gatzoulis J, Angel JC, Singh D, et al. Tibialis posterior dysfunction: a common and treatable cause of adult acquired flatfoot. BMJ. 2004; 329(7478): 1328-1333, doi: 10.1136/ bmj.329.7478.1328, indexed in Pubmed: 15576744.

14. Kokubo $T$, Hashimoto $T$, Nagura $T$, et al. Effect of the posterior tibial and peroneal longus on the mechanical properties of the foot arch. Foot Ankle Int. 2012; 33(4): 320-325, doi: 10.3113/ FAl.2012.0320, indexed in Pubmed: 22735204.

15. Kolovou G, Katsiki N, Pavlidis A, et al. Ageing mechanisms and associated lipid changes. Curr Vasc Pharmacol. 2014; 12(5): 682-689, indexed in Pubmed: 24350931.

16. Kurose T, Asai Y, Mori E, et al. Distribution and change of collagen types I and III and elastin in developing leg muscle in rat. Hiroshima J Med Sci. 2006; 55(3): 85-91, indexed in Pubmed: 16995494.

17. Lecker SH, Goldberg AL, Mitch WE. Protein degradation by the ubiquitin-proteasome pathway in normal and disease states. J Am Soc Nephrol. 2006; 17(7): 1807-1819, doi: 10.1681/ ASN.2006010083, indexed in Pubmed: 16738015.

18. Lexell J, Taylor CC. Variability in muscle fibre areas in whole human quadriceps muscle: effects of increasing age. J Anat. 1991; 174: 239-249, indexed in Pubmed: 2032938.
19. Louwerens JW, van Linge $B$, de Klerk LW, et al. Peroneus longus and tibialis anterior muscle activity in the stance phase. A quantified electromyographic study of 10 controls and 25 patients with chronic ankle instability. Acta Orthop Scand. 1995; 66(6): 517-523, indexed in Pubmed: 8553819.

20. Lui TH, Hau WW. Peroneus longus tendoscopy at the sole. Arthrosc Tech. 2018; 7(5): e479-e483, doi: 10.1016/j.eats.2017.12.001, indexed in Pubmed: 29868422.

21. Lui TH. Stabilization of medial longitudinal foot arch by peroneus longus transfer. Foot (Edinb). 2016; 27: 22-26, doi: 10.1016/j. foot.2016.03.001, indexed in Pubmed: 27058034.

22. Marcus RL, Addison O, Kidde JP, et al. Skeletal muscle fat infiltration: impact of age, inactivity, and exercise. J Nutr Health Aging. 2010; 14(5): 362-366, indexed in Pubmed: 20424803.

23. Mendicino RW, Orsini RC, Whitman SE, et al. Fibular groove deepening for recurrent peroneal subluxation. J Foot Ankle Surg. 2001; 40(4): 252-263, indexed in Pubmed: 11924687.

24. Menz HB. Biomechanics of the Ageing Foot and Ankle: A Mini-Review. Gerontology. 2015; 61(4): 381-388, doi: 10.1159/000368357, indexed in Pubmed: 25402236.

25. Mizel MS, Temple HT, Scranton PE, et al. Role of the peroneal tendons in the production of the deformed foot with posterior tibial tendon deficiency. Foot Ankle Int. 1999; 20(5): 285-289, doi: 10.1177/107110079902000502, indexed in Pubmed: 10353763.

26. Narici MV, Maganaris CN, Reeves ND, et al. Effect of aging on human muscle architecture. J Appl Physiol (1985). 2003; 95(6): 2229-2234, doi: 10.1152/japplphysiol.00433.2003, indexed in Pubmed: 12844499.

27. Narici MV, Maganaris CN. Plasticity of the muscle-tendon complex with disuse and aging. Exerc Sport Sci Rev. 2007; 35(3): 126-134, doi: 10.1097/jes.0b013e3180a030ec, indexed in Pubmed: 17620931.

28. Onambele GL, Narici MV, Maganaris CN. Calf muscle-tendon properties and postural balance in old age. J Appl Physiol (1985). 2006; 100(6): 2048-2056, doi: 10.1152/japplphysiol.01442.2005, indexed in Pubmed: 16455811.

29. Perera AM, Mason L, Stephens MM. The pathogenesis of hallux valgus. J Bone Joint Surg Am. 2011; 93(17): 1650-1661, doi: 10.2106/JBJS.H.01630, indexed in Pubmed: 21915581.

30. Ruggiero M, Cless D, Infantolino B. Upper and Lower Limb Muscle Architecture of a 104 Year-Old Cadaver. PLoS One. 2016; 11(12): e0162963, doi: 10.1371/journal.pone.0162963, indexed in Pubmed: 28033339.

31. Seah NE, de Magalhaes Filho CD, Petrashen AP, et al. Autophagymediated longevity is modulated by lipoprotein biogenesis. Autophagy. 2016; 12(2): 261-272, doi: 10.1080/15548627.20 15.1127464, indexed in Pubmed: 26671266.

32. Ugbolue UC, Gislason MK, Carter M, et al. Tensile properties of the transverse carpal ligament and carpal tunnel complex. Clin Biomech (Bristol, Avon). 2015; 30(7): 649-656, doi: 10.1016/j. clinbiomech.2015.05.010, indexed in Pubmed: 26048241.

33. van Haaren $E H$, van der Zwaard BC, van der Veen $A J$, et al. Effect of long-term preservation on the mechanical properties of cortical bone in goats. Acta Orthop. 2008; 79(5): 708-716, doi: 10.1080/17453670810016759, indexed in Pubmed: 18839380.

34. Watanabe K, Kitaoka HB, Fujii T, et al. Posterior tibial tendon dysfunction and flatfoot: analysis with simulated walking. Gait Posture. 2013; 37(2): 264-268, doi: 10.1016/j.gaitpost.2012.07.015, indexed in Pubmed: 22939754.

35. Yoshida Y, Marcus RL, Lastayo PC. Intramuscular adipose tissue and central activation in older adults. Muscle Nerve. 2012; 46(5): 813-816, doi: 10.1002/mus.23506, indexed in Pubmed: 23055318.

36. Yu TY, Pang JHS, Wu KPH, et al. Aging is associated with increased activities of matrix metalloproteinase- 2 and -9 in tenocytes. BMC Musculoskelet Disord. 2013; 14: 2, doi: 10.1186/1471-2474-14-2, indexed in Pubmed: 23281803. 